\title{
Transcranial Direct Current Stimulation to Modulate Brain Reactivity to Food Cues: Study Protocol of a Randomized Controlled Trial with fMRI (NeuroStim-Obesity)
}

\author{
Nastaran Malmir ${ }^{a}$, , Peyman Ghobadi-Azbari ${ }^{b, \#, ~ *, ~ M e g h e d i ~ V a r t a n i a n c, ~ R a s o u l ~ M a h d a v i f a r-~}$ \\ Khayati ${ }^{b}$, Sara Derafshe ${ }^{d}$, Michael A. Nitsche ${ }^{e}$, Masoud Nosratabadi ${ }^{f}$, Hamed Ekhtiari ${ }^{a}$ \\ a Iranian National Center for Addiction Studies (INCAS), Tehran University of Medical Sciences, Tehran, Iran \\ ${ }^{b}$ Department of Biomedical Engineering, Shahed University, Tehran, Iran \\ ${ }^{c}$ Department of Clinical Psychology, Tehran University, Iran \\ ${ }^{d}$ Department of Food Sciences and Technology, Islamic Azad University, Science and Research Branch, Tehran, Iran \\ ${ }^{e}$ Department of Psychology and Neurosciences, Leibniz Research Centre for Working Environment and Human \\ Factors, Germany \\ ${ }^{f}$ Head of Research Department, Paarand Center, Tehran, Iran \\ \# Authors have contributed equally as the first author
}

\begin{abstract}
Background: Obesity is one of the most serious public health concerns worldwide. Considering the multifactorial nature and increasing prevalence of obesity, many techniques have been proposed for its treatment, ranging from behavioral interventions to surgeries, but they are often ineffective, invasive, and costly. Furthermore, relapse and weight regain are common. Noninvasive brain stimulation techniques hold promise for modulating food-related brain processes and functions in these patients, potentially through increasing top-down control in response to food cue that supports reduction in calorie intake. Previous findings revealed that transcranial direct current stimulation (tDCS) over dorsolateral prefrontal cortex (DLPFC), a target site related to executive functions that support cognitive control of food craving, modulates the rewardcontrol balance towards facilitation of cognitive control and possibly suppression of rewardrelated mechanisms that drive food cue-induced craving. However, understanding the neurocognitive mechanisms of tDCS over DLPFC on the brain networks of food craving remains
\end{abstract}

\footnotetext{
* Corresponding author at: Shahed University, opposite Holy shrine of Imam Khomeini, Khalij Fars Expressway, Tehran, Iran.

E-mail address: peymanbiom@gmail.com (Peyman Ghobadi-Azbari)
} 
largely unknown. Thus, an experimental clinical trial investigating the modulatory effects of tDCS over food cue-reactivity seems promising.

Method: The NeuroStim-Obesity trial is a prospective, randomized, sham-controlled, doubleblind single session tDCS trial targeting food craving in those with obesity. Once randomized, a total of 64 subjects (age range 18-61 years) with obesity (Body Mass Index (BMI) between 25-35 $\mathrm{kg} / \mathrm{m}^{2}$ ) complete one session in which they receive either active or sham tDCS over the DLPFC. Active tDCS consisted of $2 \mathrm{~mA}$ of current applied continuously for 20 minutes with the anode placed over F4 and the cathode over F3 according to the 10-20 EEG system. The primary outcome is change in neural response to the food cue-reactivity task in the ventral striatum after a single session bilateral tDCS compared to sham stimulation. Secondary outcomes include changes in food craving evaluated by Food Craving Questionnaire-State (FCQ-S). We will also explore the predictive role of brain structural and functional networks assessed by structural, and functional magnetic resonance imaging (MRI) during both task performance and the resting-state that are acquired pre- and post-intervention to predict response to tDCS.

Discussion: The results will provide novel insight into neuroscience for the efficacy of tDCS and will advance the field towards precision medicine for obesity. Exploratory results will examine the potential predictive biomarkers for tDCS response, and eventually to provide personalized intervention for treatment of obesity.

Trial registration: This trial was retrospectively registered at Iranian Registry of Clinical Trials (IRCT) Identifier: IRCT20121020011172N4 at June 4, 2020, https://www.irct.ir/trial/45482. This protocol was prepared in accordance with the SPIRIT guidelines (Chan et al., 2013a, 2013b).

Funding: Funding for this study was provided by the "Cognitive Science and Technologies Council (CSTC) of Iran" to Masoud Nosratabadi at University of Social Welfare and Rehabilitation, Tehran, Iran.

Keywords: Obesity, Food craving, Transcranial direct current stimulation (tDCS), Functional magnetic resonance imaging (fMRI), Diffusion tensor imaging (DTI), Dorsolateral prefrontal cortex (DLPFC). 


\section{Introduction}

Obesity is a major public health concern that is characterized by overeating leading to weight gain and is often associated with metabolic syndrome, cardiovascular diseases, and psychological and psychiatric consequences (Aggoun, 2007; Bray, 2004; Kim and Popkin, 2006). According to a recent study estimating global trends in the prevalence of overweight and obesity, 105 million people were living with obesity in 1975 , increased to nearly six-fold over the past 39 years to $6 \leqslant 1$ million by $201 \leqslant$ (Di Cesare et al., 2016). Food craving, the core component of overeating in obese people, is an intense desire or urge to consume high calorie foods (Ronzio, 2003). Such craving is a form of food cue-reactivity: a conditioned appetitive response to food cue that is mostly accompanied by increased salivation (Rogers and Hill, 1989) and neural activity in gustatory and reward-relevant brain areas (Simmons et al., 2005). As such, food craving and other forms of food cue reactivity may function as conditioned responses that that serve as triggers for increased

food consumption (Larsen et al., 2012) and weight gain (Murdaugh et al., 2012), which may exacerbate the risk for rising obesity rates (Morris et al., 2015). Albeit there are positive outcomes in controlling obesity using nutritional, psychological and pharmacological approaches, a majority of patients with frequent food cravings manifest a chronic, relapsing course of disease within 1 year of obesity treatments (Anton, 1999; Budak and Thomas, 2009; Odom et al., 2010) resulting in a need for alternative or adjuvant treatment options. Transcranial direct current stimulation (tDCS), a simple, cheap, safe, and non-invasive brain stimulation method established during last two decades in the human neuroscience research has been proposed as a potential adjuvant in food craving and obesity management programs.

According to the personalized medicine frameworks, future obesity research seeks to relate obesity disorders to more distinct pathophysiological and neurobehavioral entities, e.g. to paradigms of dysfunctional neural networks associated with cognitive control, reward sensitivity, and conditioning resulting in resistance to homoeostatic signals (Higgs et al., 2017; Kullmann et al., 2015; Lowe et al., 2019; Volkow et al., 2011). This notion supports novel interventions in terms of modulating dysregulated networks and neural activity. Accordingly, as shown in the recent neuroimaging studies, the use of non-invasive brain stimulation technologies in neurology and psychiatry disorders, may provide potentials to modulate dysregulated networks and neural 
activity. Among the existing non-invasive stimulation technologies, tDCS offers a generally acceptable safety, tolerability, ease of use, cost, and portability, which may make it as a treatment approach in different neurological and psychiatric disorders (Bikson et al., 2016). The therapeutic potential of tDCS intervention for obesity is hypothesized to be based on the modulation of dysregulated top-down control by prefrontal cortex (PFC) function and rewardprocessing in subcortical limbic structures, mainly ventral striatum. In recent years, data derived from neuroimaging and cognitive assessments provide evidence that food cue-reactivity, particularly in obese persons, is associated with dysregulated activities within the prefrontal regions, specifically, the dorsolateral prefrontal cortex (DLPFC). The potential for DLPFC neuromodulation might remediate these dysregulated activities within prefrontal brain regions that has been associated with both impaired inhibitory control (i.e., binge eating and purging) and poor cognitive flexibility (e.g., the obsessive concerns with eating, weight, and shape) (Hall et al., 2017; Lowe et al., 2017; McClelland et al., 2016; Mostafavi et al., 2018).

Accordingly, therapeutic strategies that modulate brain activity in the DLPFC might regulate or suppress food cue-reactivity, cravings and appetite. Examining the effect of cortical activity modulation using high-frequency rTMS over the left DLPFC in eating disorders and obesity was tested in many studies and a temporary anti-craving effect of this modulation has been demonstrated (Uher et al., 2005; Van Den Eynde et al., 2013). Another clinical study also reported beneficial effects of using high-frequency rTMS applied over the left DLPFC to modify cue-induced food cravings and binge eating in people with a bulimic eating disorder (Van den Eynde et al., 2010). This finding was replicated using bilateral tDCS to the DLPFC (anode over F4/cathode over F3), which demonstrate a reduction in self-reported cravings for active stimulation as compared to sham stimulation (Fregni et al., 2008; Goldman et al., 2011; Kekic et al., 2014).

A recent meta-analysis regarding the effect of DLPFC $t D C S$ as a potential therapeutic modality for food craving in 15 RCTs found a significant effect size supporting active tDCS, compared to sham stimulation in terms of reduction of food craving and energy intake (Mostafavi et al., 2018). One other meta-analysis over 17 RCTs came to the same result (Jansen et al., 2013), however, another meta-analysis over 11 RCTs has not reported the same effect of tDCS in regards to food cravings 
and consumption (Lowe et al., 2017), which can be attributed to methodological variations in data analyses and the scope of the review.

However, no study has evaluated the neurofunctional mechanisms of action of tDCS on food cuereactivity. Therefore, the present study was designed as a randomized parallel-design, shamcontrolled, double-blind trial to investigate the neurofunctional mechanisms of action for tDCS to modulate food cue-reactivity and cravings in those with obesity using fMRI $(n=64)$. A single session bilateral tDCS over the DLPFC will be applied in half of the subjects while the other half will receive sham stimulation. Effects on eating behavior as well as on brain function will be assessed at multiple time-points using both task-based and rest functional neuroimaging modalities. The current protocol describes the design and methods implemented in the NeuroStim-Obesity study.

\section{Study Design and Methods}

\subsection{Design and Setting}

NeuroStim-Obesity is a single-center trial, which will be conducted the National Brain Mapping Lab, Tehran, Iran. The study will be conducted according to a double-blind randomized shamcontrolled design. A total of 64 people with obesity between the age of 18 and 61 years will be randomly divided into two parallel arms which deliver active or sham DLPFC tDCS.

The course of the trial is visualized in Figure 1. We have prepared a video about how to execute the project and details of project process that the subjects can watch it before testing day and they are encouraged to share their questions or concerns. After signing the consent form, eligible participants will undergo intensive baseline assessments using self-report and clinical assessments. Participants in the active arm will receive $2 \mathrm{~mA}$ anodal tDCS for 20 min over the Right DLPFC with the cathode over the Left DLPFC. The set-up for sham and real stimulation sessions are exactly the same. Structural, resting-state (rs) and task-based functional MRI (pictorial food cue exposure) will be done immediately before and after active or sham tDCS. Immediate craving will be assessed with a single item 0-100 VAS question at multiple time-points before and after imaging and tDCS. The Ethics Committee of Iran University of Medical Sciences approved the research to trial (IR.IUMS.REC.1396.0459), which is registered with the Iranian Registry of Clinical Trials (IRCT20121020011172N4). 


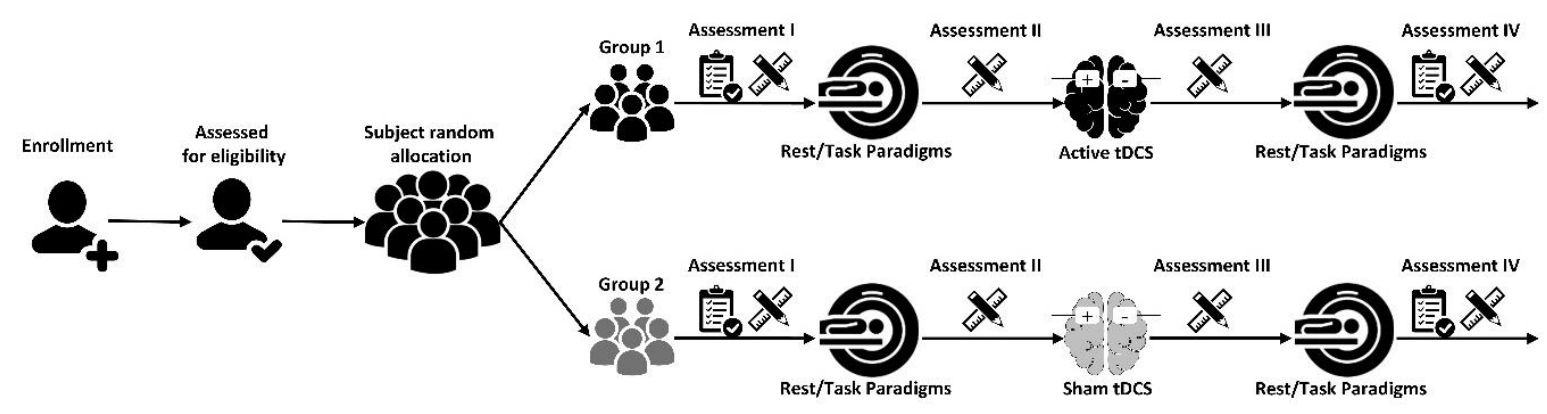

FIGURE 1 | NeuroStim-Obesity flowchart.

\subsection{Participants}

We plan to enroll 64 subjects from specialized nutrition clinics by flyer advertising. We also made a video to get people more familiar with our project. Participants must follow all of the inclusion criteria below:

1. Age $\geq 18$ and $<61$ years old.

2. Right-handedness.

3. Persian speaking.

4. BMI: $25-35 \mathrm{~kg} / \mathrm{m}^{2}$.

5. Frequent food cravings ( $\geq 3$ per day during last month, as assessed by self-report questionnaire).

6. Being responsive in food cue-reactivity screening at baseline (defined as mean craving scores (> 70) in food-related images on the Visual Analogue Scale 0-100).

The participant with any of the following criteria will be excluded from the study:

1. Unwillingness or inability to complete any of the major aspects of the study protocol including food cue rating, or behavioral assessments.

2. Comorbid psychiatric disorders (i.e., depressive, bipolar, or psychotic disorders), which is evaluated by a mental health specialist.

3. Active suicidal ideation with intent or plan as determined by self-report or assessment by a mental health specialist during the initial screening or any other phase of the study.

4. Unstable medical disorder reported in subject's medical history or by a clinician assessment.

5. Non-correctable vision or hearing problems.

6. Personal or family history of seizures. 
7. History of stroke.

8. Any other condition the research team feel would put the subject at risk for entering the study.

9. Contraindication to tDCS (pacemaker, a metal embedded in the scalp or brain, skin lesions at the site of stimulation, and history of head injury or neurosurgery) and MRI (claustrophobia, metallic implants, ferromagnetic metals in the scalp or brain, and pregnant women).

If all eligibility criteria are reached and participants provide written informed consent for study participation, they will be included in the study sample. Eligibility will be determined by the trained study staff during screening and baseline assessments.

\subsection{Intervention}

Transcranial direct current stimulation will be administered via a battery-driven stimulator (DCStimulator Plus, Neuroconn GmbH, Germany). Direct current will be transferred by two salinesoaked sponge electrodes $\left(7 \times 5 \mathrm{~cm}^{2}\right)$. For effective bilateral tDCS, the tDCS montage will comprise placement of the anode over the right DLPFC and the cathode over the left DLPFC which corresponds to the F4 and F3 areas, according to the 10-20 EEG system. Active tDCS will be delivered with a constant current of $2 \mathrm{~mA}$ for 20 minutes (ramp-up/down: 30 seconds). The montage and dose of stimulation were chosen based on the previous studies for food craving in healthy adults (Fregni et al., 2008; Goldman et al., 2011; Kekic et al., 2014). For the sham group, the same electrode position and ramp-up/down time will be used, but the $2 \mathrm{~mA}$ current will be delivered only during the first $1 \mathrm{~min}$ of the 20-minute stimulation period to elicit a transient tingling sensation on the scalp and to blind subjects as to the respective stimulation condition. Participants will be told about a mild tingling and itching sensation under the electrodes during the stimulation. After each active or sham-tDCS session, participants will complete an adverseevents questionnaire (Antal et al., 2017).

\subsection{Outcomes Measures and Assessments}

Outcome measures for the food cue-reactivity task (see Figure 2) will be acquired pre- and post$\mathrm{fMRI}$ at each session. At pre- and post-assessments, additional outcome measures targeting neural substrates, will be assessed. The timeline of assessment measures is shown in Table 1. 
TABLE 1 | Schedule of enrollment, interventions, and assessments.

\begin{tabular}{|c|c|c|c|c|c|c|c|c|c|c|}
\hline \multirow[b]{4}{*}{ Time point } & \multirow[b]{4}{*}{ Measurement } & \multirow[b]{4}{*}{ Mode } & \multicolumn{8}{|c|}{ Post-allocation } \\
\hline & & & \multirow{2}{*}{$\begin{array}{c}\text { Baseline } \\
\sim 1 \mathrm{~h}\end{array}$} & \multirow{2}{*}{$\begin{array}{c}\text { PrefMrI1 } \\
\sim 10 \text { min }\end{array}$} & \multirow{2}{*}{$\begin{array}{c}\text { fMRI } \\
\sim 30 \mathrm{~min}\end{array}$} & \multirow{2}{*}{$\begin{array}{l}\text { Post }_{\mathrm{fMRI1}} \\
\sim 10 \mathrm{~min}\end{array}$} & \multirow{2}{*}{$\begin{array}{c}\text { Intervention } \\
30 \mathrm{~min}\end{array}$} & \multirow{2}{*}{$\begin{array}{c}\text { Pre }_{\text {fMRI2 }} \\
\sim 10 \text { min }\end{array}$} & \multirow{2}{*}{$\begin{array}{c}\mathrm{fMRI_{2 }} \\
\sim 30 \mathrm{~min}\end{array}$} & \multirow{3}{*}{$\begin{array}{c}\text { Post }_{\mathrm{fMRI}} \\
\sim 25 \mathrm{~min} \\
\text { T7 }\end{array}$} \\
\hline & & & & & & & & & & \\
\hline & & & TO & T1 & T2 & T3 & T4 & T5 & T6 & \\
\hline \multicolumn{11}{|l|}{ Enrollment } \\
\hline Eligibility screening & & Paper & $x$ & & & & & & & \\
\hline Informed consent & & Paper & $x$ & & & & & & & \\
\hline Neuropsychological & Demographic data & Paper & $\mathrm{x}$ & & & & & & & \\
\hline \multirow[t]{6}{*}{ Screening } & EHI (Oldfield, 1971) & Paper & $\mathrm{X}$ & & & & & & & \\
\hline & CES (Mostafavi et al., 2016) & Paper & $\mathrm{X}$ & & & & & & & \\
\hline & DASS-21 (Sahebi et al., 2005) & Paper & $\mathrm{X}$ & & & & & & & \\
\hline & TFEQ-R-18 (Mostafavi et al., 2017) & Paper & $x$ & & & & & & & \\
\hline & EDDS (Stice et al., 2000) & Paper & $x$ & & & & & & & \\
\hline & Food craving pictures & Tablet-PC & $x$ & & & & & & & \\
\hline Intervention & & & & & & & $\leftrightarrow$ & & & \\
\hline Viewing Task & Food cue-reactivity & Computer & & & $\mathrm{X}$ & & & & $\mathrm{X}$ & \\
\hline Brain stimulation & tDCS (active vs. sham) & Device & & & & & $x$ & & & \\
\hline \multirow[t]{6}{*}{ Questionnaires } & FCQ-T (Kachooei and Ashrafi, 2016) & Paper & & $x$ & & & & & & $x$ \\
\hline & FCQ-S (Cepeda-Benito et al., 2000) & Paper & & $x$ & & & & & & $x$ \\
\hline & Self-reported craving (VAS) & Paper & & $\mathrm{X}$ & & $\mathrm{X}$ & & $\mathrm{X}$ & & $\mathrm{X}$ \\
\hline & Baseline hunger (VAS) & Paper & & $x$ & & $x$ & & $x$ & & $x$ \\
\hline & Seven affective states (VAS) & Paper & & $\mathrm{X}$ & & $\mathrm{X}$ & & $X$ & & $\mathrm{X}$ \\
\hline & Blindness & Paper & & & & & & & & $\mathrm{X}$ \\
\hline
\end{tabular}




\begin{tabular}{llll} 
& & & \\
\cline { 2 - 4 } & \multicolumn{1}{l}{ Sensations related to tES (Antal et al., 2017) } & Paper & \\
\hline Additional Assessments & & Computer & $\mathrm{X}$ \\
\hline Physical measures & $\mathrm{fMRI}$ & Computer & $\mathrm{X}$ \\
\cline { 2 - 4 } & $\mathrm{DTI}$ & & \\
\hline
\end{tabular}

Abbreviations: EHI, Edinburgh Handedness Inventory; CES, Compulsive Eating Scale; DASS-21, Depression Anxiety Stress Scales-21; TFEQ-R18, Three-Factor Eating

Questionnaire-R18; EDDS, Eating Disorder Diagnostic Scale; FCQ-T, Food Craving Questionnaire-Trait; FCQ-S, Food Craving Questionnaire-State; VAS, Visual Analog Scale; DTI, Diffusion Tensor Imaging.

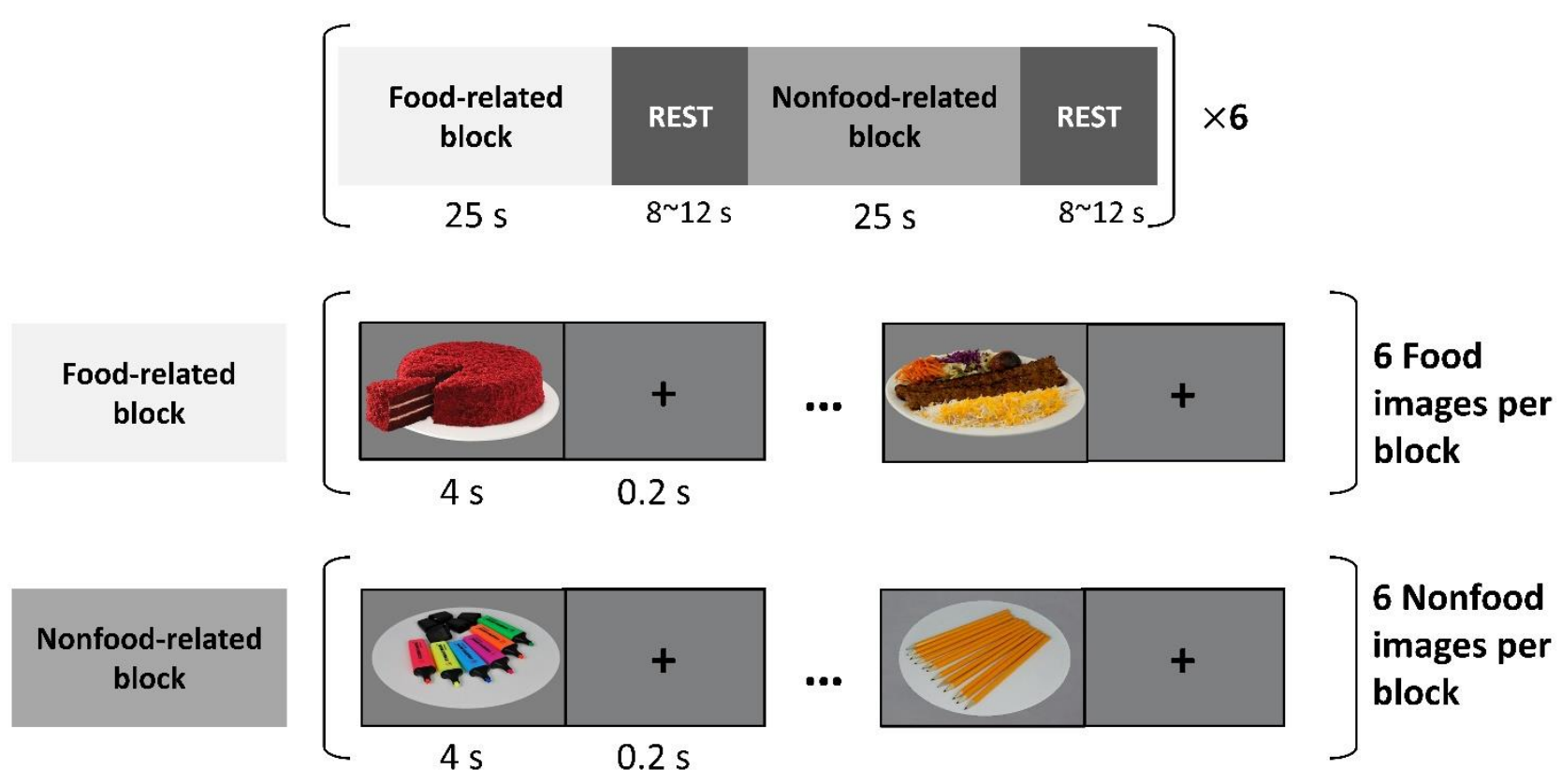

FIGURE 2 | Structure of the food cue-reactivity task. The task included food blocks and non-food blocks. 
Each outcome measure will be analyzed regarding potential comparisons between the study groups (active vs. sham tDCS).

\subsubsection{Primary Outcomes}

The primary outcome will be neural response to food cue exposure in the ventral striatum region of interest, as assessed by the BOLD signal changes with voxel-wise analysis in the ventral striatum from before to after intervention. Given the hypothesis of this study, another primary outcome measure is the changes from pre-fMRI to post-fMRI in VAS scores (for food craving) at post-tDCS compared to changes at pre-tDCS in the sham and active tDCS group.

\subsubsection{Secondary Outcomes}

(a) Change in FCQ-S scores from before tDCS to after tDCS, as measured by Food Craving Questionnaire-State (Cepeda-Benito et al., 2000).

(b) Change in resting-state functional connectivity between cortical and subcortical regions, as assessed by correlation between spontaneous BOLD signal fluctuations in subcortical ROIs and voxels within prefrontal cortex and Insula from before tDCS to after tDCS.

(c) Change in resting-state functional connectivity under the stimulated area, as assessed by correlation between spontaneous BOLD signal fluctuations in the cortical area under the Anode/Cathode electrodes and whole brain from before tDCS to after tDCS.

(d) Change in task-based functional activation in prefrontal cortical areas and subcorticallimbic areas, as assessed by the BOLD signal changes with voxel-wise analysis in the regions of interests (ROIs) (prefrontal cortex, insula, thalamus, and extended amygdala) from before tDCS to after tDCS.

(e) Change in task-based functional connectivity between cortical and subcortical regions, as assessed by psychophysiological interaction (PPI) between spontaneous BOLD signal fluctuations in subcortical ROIs and voxels within prefrontal cortex and Insula from before tDCS to after tDCS.

(f) Change in task-based functional connectivity under the stimulated area, as assessed by psychophysiological interaction (PPI) between spontaneous BOLD signal fluctuations in 
the cortical area under the Anode/Cathode electrodes and whole brain from before tDCS to after tDCS.

(g) Change in Resource Allocation Index (RAI) in rs-fMRI, as assessed by correlation among default mode network (DMN), saliency network (SN) and executive control network (ECN) in rs-fMRI from before tDCS to after tDCS (Lerman et al., 2014).

\subsubsection{Exploratory Outcomes}

Exploratory analyses will be conducted for measures of subjective, behavioral, neuropsychological, or neural circuits which will be analyzed for identifying potential predictors of food cue-reactivity task performance, and responsiveness to the intervention, as measures by an exploratory regularized regression model. Additionally, the induced E-field in the prefrontal area, derived from analysis of computational finite element model, will be included as potential predictor of neural response to tDCS (Esmaeilpour et al., 2019; Ghobadi-Azbari et al., 2020).

\subsection{Outcome Assessments}

The study timeline shown in Table 1 provides an overview of the time schedule of enrolment, interventions, and assessments.

TABLE 2 | MRI data acquisition parameters.

\begin{tabular}{|c|c|}
\hline Sequence & Main parameters \\
\hline Resting-state fMRI & $\begin{array}{l}T R=2500 \mathrm{~ms}, \text { TE }=30 \mathrm{~ms}, \mathrm{FOV}=192 \times 192 \mathrm{~mm}^{2}, 43 \text { slices, } 144 \text { volumes, } 3.0 \times \\
3.0 \times 3.0 \mathrm{~mm}^{3} \text {, flip angle }=90^{\circ}\end{array}$ \\
\hline Task-based fMRI & $\begin{array}{l}T R=2500 \mathrm{~ms}, T E=23 \mathrm{~ms}, \mathrm{FOV}=192 \times 192 \mathrm{~mm}^{2}, 43 \text { slices, } 167 \text { volumes, } 3.0 \times \\
3.0 \times 3.0 \mathrm{~mm}^{3} \text {, flip angle }=70^{\circ}\end{array}$ \\
\hline DTI & $\begin{array}{l}T R=8000 \mathrm{~ms}, T E=68 \mathrm{~ms}, 70 \text { slices, } 2 \times 2 \times 2 \mathrm{~mm}^{3}, 64 \text { directions }(b=1000 \\
\left.\mathrm{s} / \mathrm{mm}^{2}\right)\end{array}$ \\
\hline T1w & $\begin{array}{l}T R=1810 \mathrm{~ms}, T E=3.45 \mathrm{~ms}, \mathrm{TI}=1100 \mathrm{~ms}, 176 \text { slices, } 1.03 \times 1.03 \times 1.0 \mathrm{~mm}^{3} \text {, flip } \\
\text { angle }=7^{\circ}\end{array}$ \\
\hline $\mathrm{T} 2 \mathrm{w}$ & $T R=3200 \mathrm{~ms}, T E=408 \mathrm{~ms}, 176$ slices, $0.45 \times 0.45 \times 0.9 \mathrm{~mm}^{3}$, flip angle $=120^{\circ}$ \\
\hline
\end{tabular}

$\mathrm{TR}$, repetition time; TE, echo time; TI, inversion time; FOV, field of view; fMRI, functional magnetic resonance imaging; DTI, diffusion tensor imaging 


\subsubsection{Baseline Assessments}

At baseline assessment (TO), participants will be given written informed consent, and complete a demographic questionnaire, a handedness inventory (Oldfield, 1971), Depression Anxiety Stress Scales-21 (DASS-21; (Sahebi et al., 2005)), Eating Disorder Diagnostic Scale (EDDS; (Stice et al., 2000)), Compulsive Eating Scale (CES; (Mostafavi et al., 2016)), Three-Factor Eating Questionnaire-R18 (TFEQ-R18; (Mostafavi et al., 2017)). Afterward, participants will perform the

food cue-reactivity training task to learn about the actual fMRI task (see section on MRI below), that consists of one practice trial with 6 images of food and non-food products. The baseline assessment will take approximately $1 \mathrm{~h}$.

\subsubsection{Pre- and Post-Assessments}

The $0-100$ continuous VAS will provide the possibility for assessing the self-reported food craving, hunger, and affective states in multiple time points before and after fMRI and tDCS (T1, T3, T5, and T7). Furthermore, FCQ-S and FCQ-T will provide the possibility for assessing the intensity of trait and state dimensions of food cravings before the first fMRI (T1: pre tDCS) and after the second fMRI (T7: Post tDCS). Finally, participants will complete a questionnaire to assess blindness and potential adverse-events of tDCS (T7).

\subsection{Magnetic Resonance Imaging}

MRI will be assessed at the Iranian National Brain Mapping Laboratory with a 3 Tesla scanner (Siemens Prisma) using a 20-channel head coil, prior to and immediately after intervention (see Table 2 for MRI data acquisition parameters).

Both MRI sessions include structural T1-weighted acquisition as well as resting-state $\mathrm{fMRI}$ to assess functional connectivity within and between large-scale networks that mediate food craving. Furthermore, a food cue-reactivity fMRI task will be used to assess intervention associated changes in prefrontal functioning (see Figure 2). At the end of the post-tDCS MRI assessment, additional T1- and T2-weighted images will be acquired with parameters optimized 
for computational finite-element method (FEM) modeling to calculate electric field strength and distribution induced by tDCS (Thielscher et al., 2015; Windhoff et al., 2013), see Figure 3 for sample modeling analysis. After the MRI sessions, a separate DTI scan will be also performed to assess the structural integrity of the cortical-subcortical tracts, via quantification of fractional anisotropy (Pierpaoli and Basser, 1996). In the food cue-reactivity task, participants will view 12 blocks of 6 images each ( 6 blocks with foods and 6 blocks with non-foods). Each block consists of 6 images of either food or neutral valence presented for 4000 milli seconds (ms) with an interstimulus interval of $200 \mathrm{~ms}$ followed by an inter-block interval (i.e., gray screen with crosshair) with a duration (8-12 seconds). They will be given the following task instruction: "In the next task you will see food and non-food products. Please look at the images and pay close attention, since during the MRI session your job will be to press a button on the scroll wheel whenever you see the yellow border images."
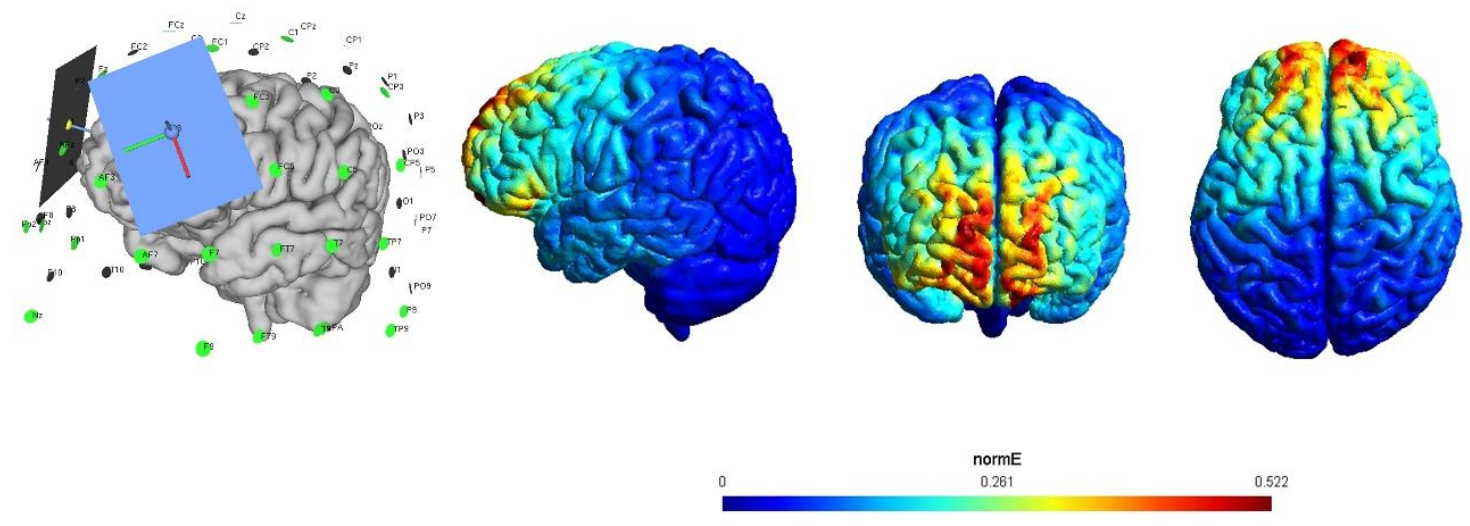

FIGURE 3 | Electrode positioning (anode, F4; cathode, F3) and computational model of the E-field induced in tDCS using SimNIBS, for one example participant ( $f, 27$ years).

\subsection{Safety Outcome}

Subjects will be fully informed about the foreseeable risks and discomforts associated with participation in this study. The consent forms describe these risks and discomforts clearly. Patients must know that they have the option to withdraw from the study at any time. Withdrawal from this study can be done without consequence. The investigators may also choose to terminate a participant from this study, if they suffer a severe adverse event, do not follow study requirements, or feel that continued participation would put the person at a greater risk 
than indicated. Headaches, itching, and paresthesia are generally very mild with tDCS and are limited to the actual treatment duration. Since skin nerves habituate to the electrical stimulation rather quickly, most subjects are not aware of the stimulation after about the first 1-minute. This is what allows sham stimulation to be effectively masked. More persistent headache can be treated with acetaminophen or ibuprofen. The range of stimulation intensities for human studies of tDCS is usually $1-2 m A m p s$ as we do in our study. Skin redness is common with tDCS studies because the electrical stimulation increases local blood flow under the electrodes. This redness should dissipate within 30-minutes or less. There should be no evidence of redness or skin breakdown before the tDCS application.

\subsection{Sample Size Estimation}

Based on recent study in the field using single-session bilateral tDCS over the DLPFC compared to sham tDCS (Goldman et al., 2011), we estimated an effect size of 0.55. To demonstrate the intervention effect in the primary outcome, 64 participants (32 per tDCS group) need to be included in the analysis with a two-group t-test with a 0.05 two-sided significance level and a power of $80 \%$. Sample size estimation was conducted using PASS software (Hintze, 2011).

\subsection{Blinding and Randomization}

During recruited process, participants are informed that the study evaluates neural responses to the transcranial direct current stimulation but are not told anything about sham tDCS and differences between them. The screen of the tDCS device is hidden from participants and the group allocation remains indistinguishable to both participants and the investigators. The sham tDCS condition consists of a $30 \mathrm{~s}$ ramp up to $2 \mathrm{~mA}$ and immediately ramp down to $0.0 \mathrm{~mA}$ over 30 s to let participants feel the itching resembling to the real stimulation. To ensure the success of blinding, we will ask participants at the end of the study to guess whether the stimulation was real or sham.

We use the sealed envelope method (see https://www.sealedenvelope.com) to group assignment (i.e., either active-tDCS or sham-tDCS) at the beginning of the study (by NM). All participants will have a unique ID during the study process and only an independent clinician who 
is not involved in the study knows them (NS). Main investigators and participants will not have any knowledge about allocation of study groups.

A blocked randomization (6-block) will be used to balance the number of participants of the two groups that are run in parallel. Each subject randomly assigns to each of active or control DLPFC tDCS.

\subsection{Quality Control and Data Management}

The research team is comprised principal investigator (HE), two professionally trained researchers (NM and PGA) and research assistances (MN, SD and NS) who have regular weekly meetings to ensure about consistency on project implementation and discuss about trial progress. The data collected in this trial will comprise neuroimaging data and psychological questionnaires. Two professionally trained researchers (NM and PGA) are responsible for collecting confirmed data and data entry.

\subsection{Statistical Methods}

Baseline participant characteristics will be presented as mean \pm standard deviation for continuous variables or percentages for categorical variables. Participant demographics data will be compared between the active tDCS and sham tDCS groups, using a two-sample t-test (Student's t-test).

To control for the tDCS intervention efficacy, a repeated-measures analysis of variance (ANOVA) will be performed on FCQ-T with time (before/after tDCS) as the within-subject factor and group (active tDCS/sham tDCS) as the between-group factor. The same strategy will be followed for behavioral and neuropsychological data.

Changes in functional neural parameters (resting state and task-related $\mathrm{fMRI}$ ) will be preprocessed and analyzed using the Analysis of Functional Neurolmages (AFNI) (Cox, 1996). To assess brain-behavior associations, the correlations between changes in brain activity/connectivity and subjective craving will be investigated using Pearson correlation test. As an exploratory analysis, the definition of major prognostic factors for response to active tDCS will 
be assessed using the exploratory regularized regression model. Assessment of the model validity will be performed using leave-one-out cross validation within this dataset and will be validated with subsequent test datasets in the future studies. All statistical analyses will be run using the $R$ Statistical Package (Team, 2013).

\section{Discussion}

This randomized controlled trial with two parallel groups will investigate the effects of a single session of bilateral tDCS on food cue-induced cravings as well as functional neural parameters in obese people with frequent food cravings. In the active group, bilateral tDCS (1 mA, $20 \mathrm{~min}$ ) will be applied over the DLPFC (anode over the right DLPFC, cathode over the left DLPFC) while the control group will receive sham tDCS (1 mA, $30 \mathrm{~s})$. Findings of this study will contribute to the understanding of predictive biomarkers, as well as the underlying mechanisms of tDCS effects in the brain response to food related cues.

As discussed previously, prior studies indicated that prefrontal cortex tDCS modulated food cravings (Fregni et al., 2008; Goldman et al., 2011; Kekic et al., 2014), and provided evidence that food craving is associated with DLPFC activity. The mechanistic neural substrates by which DLPFC stimulation decreases food cravings are unknown, although data suggest that the amount of activity in the right prefrontal cortex may determine the degree of inhibition over downstream circuits that promote overeating (Alonso-Alonso and Pascual-Leone, 2007). On the one hand this trial aims to demonstrate the mechanistic neural substrates of tDCS in people with obesity. Resting state and task-based fMRI will presumably allow us to examine mechanistic hypotheses for the modulatory role for prefrontal tDCS in both small scale top-down regulation and largescale network interactions. On the other hand, this study should make a contribution to the identification of predictive biomarkers of response to tDCS. Further explorations using machine learning methods concerning the personalized interventions will help to identify prognostic biomarkers of tDCS response aiming to improve tDCS methods in the individual level and to understand the mechanisms of action of tDCS on a predictive basis. 
In conclusion, the current clinical trial will investigate how brain functional neural parameters at the network level may influence tDCS impact on food cue-reactivity and craving, and also address how the tDCS can modulate brain function with the hope of improving treatment outcome. Our study will provide new insights for the neuromodulatory treatments for obesity, by identifying functional activity/connectivity biomarkers of the clinical response to tDCS stimulation and hopefully contribute significantly to refine this method to allow the customization of therapeutic protocols in the individual level.

\section{Trial Status}

Enrolment for this study began in July 2019. At the time of submission, we have enrolled 30 participants till June 30, 2020.

\section{Abbreviations}

tDCS: Transcranial Direct Current Stimulation; DLPFC: Dorsolateral Prefrontal Cortex; BMI: Body Mass Index; fMRI: Functional Magnetic Resonance Imaging; IRCT: Iranian Registry of Clinical Trials; DTI: Diffusion Tensor Imaging; VAS: Visual Analogue Scale; ROI: Regions of Interest; PPI: Psychophysiological Interaction; RAI: Resource Allocation Index; DMN: Default Mode Network; SN: Saliency Network; ECN: Executive Control Network; DASS-21: Depression Anxiety Stress Scales-21; FCQ-T: Food Craving Questionnaire-Trait; FCQ-S: Food Craving Questionnaire-State; CES: Compulsive Eating Scale; TFEQ-R18: Three-Factor Eating Questionnaire-R18; FEM: FiniteElement Method; ANOVA: Analysis Of Variance; AFNI: Analysis of Functional Neuroimages; CSTC: Cognitive Science And Technologies Council. 


\section{Acknowledgements}

Authors would like to thank Mohammad Reza Ay, Gholam-Ali Hossein-Zadeh, Narges Sadeghbeigi, Mahdi Ghiyasi, and Shaghayegh Karimi for their valuable thoughts, comments and helps.

\section{Availability of Data and Materials}

Not applicable.

\section{Competing Interests}

The authors declare that they have no competing interests.

\section{Consent for Publication}

Not applicable.

\section{Ethics approval and consent to participate}

This study is approved by the by ethics committee of research, Iran University of Medical Science (IR.IUMS.REC.1396.0459) and registered at IRCT (IRCT20121020011172N4). All subjects fully informed about the project and patients must know that they have the option to withdraw from the study at any time. Withdrawal from this study can be done without consequence. Any adverse effect during the study will be recorded in detail. Findings will be published in peerreviewed journals and academic conferences regardless of results. 


\section{References}

Aggoun, Y., 2007. Obesity, Metabolic Syndrome, and Cardiovascular Disease. https://doi.org/10.1203/pdr.0b013e31805d8a8c

Alonso-Alonso, M., Pascual-Leone, A., 2007. The right brain hypothesis for obesity. J. Am. Med. Assoc. https://doi.org/10.1001/jama.297.16.1819

Antal, A., Alekseichuk, I., Bikson, M., Brockmöller, J., Brunoni, A.R., Chen, R., Cohen, L.G., Dowthwaite, G., Ellrich, J., Flöel, A., Fregni, F., George, M.S., Hamilton, R., Haueisen, J., Herrmann, C.S., Hummel, F.C., Lefaucheur, J.P., Liebetanz, D., Loo, C.K., McCaig, C.D., Miniussi, C., Miranda, P.C., Moliadze, V., Nitsche, M.A., Nowak, R., Padberg, F., Pascual-Leone, A., Poppendieck, W., Priori, A., Rossi, S., Rossini, P.M., Rothwell, J., Rueger, M.A., Ruffini, G., Schellhorn, K., Siebner, H.R., Ugawa, Y., Wexler, A., Ziemann, U., Hallett, M., Paulus, W., 2017. Low intensity transcranial electric stimulation: Safety, ethical, legal regulatory and application guidelines. Clin. Neurophysiol. https://doi.org/10.1016/j.clinph.2017.06.001

Anton, R.F., 1999. What is craving? Models and implications for treatment. Alcohol Res. Heal. 23, 165173.

Bikson, M., Grossman, P., Thomas, C., Zannou, A.L., Jiang, J., Adnan, T., Mourdoukoutas, A.P., Kronberg, G., Truong, D., Boggio, P., Brunoni, A.R., Charvet, L., Fregni, F., Fritsch, B., Gillick, B., Hamilton, R.H., Hampstead, B.M., Jankord, R., Kirton, A., Knotkova, H., Liebetanz, D., Liu, A., Loo, C., Nitsche, M.A., Reis, J., Richardson, J.D., Rotenberg, A., Turkeltaub, P.E., Woods, A.J., 2016. Safety of Transcranial Direct Current Stimulation: Evidence Based Update 2016. Brain Stimul. 9, 641-661. https://doi.org/10.1016/j.brs.2016.06.004

Bray, G.A., 2004. Medical Consequences of Obesity. J. Clin. Endocrinol. Metab. 89, 2583-2589. https://doi.org/10.1210/jc.2004-0535

Budak, A.R., Thomas, S.E., 2009. Food Craving as a Predictor of "Relapse" in the Bariatric Surgery Population: A Review with Suggestions. Bariatr. Nurs. Surg. Patient Care 4, 115-121. https://doi.org/10.1089/bar.2009.9979

Cepeda-Benito, A., Gleaves, D.H., Fernández, M.C., Vila, J., Williams, T.L., Reynoso, J., 2000. The development and validation of Spanish versions of the State and Trait Food Cravings Questionnaires. Behav. Res. Ther. 38, 1125-1138. https://doi.org/10.1016/S0005-7967(99)00141-2

Chan, A.W., Tetzlaff, J.M., Altman, D.G., Laupacis, A., Gøtzsche, P.C., Krleža-Jerić, K., Hróbjartsson, A., Mann, H., Dickersin, K., Berlin, J.A., Doré, C.J., Parulekar, W.R., Summerskill, W.S.M., Groves, T., Schulz, K.F., Sox, H.C., Rockhold, F.W., Rennie, D., Moher, D., 2013a. SPIRIT 2013 statement: Defining standard protocol items for clinical trials. Ann. Intern. Med. https://doi.org/10.7326/00034819-158-3-201302050-00583

Chan, A.W., Tetzlaff, J.M., Gøtzsche, P.C., Altman, D.G., Mann, H., Berlin, J.A., Dickersin, K., Hróbjartsson, A., Schulz, K.F., Parulekar, W.R., Krleza-Jeric, K., Laupacis, A., Moher, D., 2013b. SPIRIT 2013 explanation and elaboration: guidance for protocols of clinical trials. BMJ 346. https://doi.org/10.1136/bmj.e7586

Cox, R.W., 1996. AFNI: Software for analysis and visualization of functional magnetic resonance neuroimages. Comput. Biomed. Res. 29, 162-173. https://doi.org/10.1006/cbmr.1996.0014 
Di Cesare, M., Bentham, J., Stevens, G.A., Zhou, B., Danaei, G., Lu, Y., Bixby, H., Cowan, M.J., Riley, L.M., Hajifathalian, K., Fortunato, L., Taddei, C., Bennett, J.E., Ikeda, N., Khang, Y.H., Kyobutungi, C., Laxmaiah, A., Li, Y., Lin, H.H., Miranda, J.J., Mostafa, A., Turley, M.L., Paciorek, C.J., Gunter, M., Ezzati, M., Abdeen, Z.A., Hamid, Z.A., Abu-Rmeileh, N.M., Acosta-Cazares, B., Adams, R., Aekplakorn, W., Aguilar-Salinas, C.A., Ahmadvand, A., Ahrens, W., Ali, M.M., Alkerwi, A., AlvarezPedrerol, M., Aly, E., Amouyel, P., Amuzu, A., Andersen, L.B., Anderssen, S.A., Andrade, D.S., Anjana, R.M., Aounallah-Skhiri, H., Ariansen, I., Aris, T., Arlappa, N., Arveiler, D., Assah, F.K., Avdicová, M., Azizi, F., Babu, B. V., Balakrishna, N., Bandosz, P., Banegas, J.R., Barbagallo, C.M., Barceló, A., Barkat, A., Barros, M. V., Bata, I., Batieha, A.M., Batista, R.L., Baur, L.A., Beaglehole, R., Romdhane, H. Ben, Benet, M., Bernabe-Ortiz, A., Bernotiene, G., Bettiol, H., Bhagyalaxmi, A., Bharadwaj, S., Bhargava, S.K., Bhatti, Z., Bhutta, Z.A., Bi, H.S., Bi, Y., Bjerregaard, P., Bjertness, E., Bjertness, M.B., Björkelund, C., Blake, M., Blokstra, A., Bo, S., Bobak, M., Boddy, L.M., Boehm, B.O., Boeing, H., Boissonnet, C.P., Bongard, V., Bovet, P., Braeckman, L., Bragt, M.C.E., Brajkovich, I., Branca, F., Breckenkamp, J., Brenner, H., Brewster, L.M., Brian, G.R., Bruno, G., Bueno-DeMesquita, H.B., Bugge, A., Burns, C., De León, A.C., Cacciottolo, J., Cama, T., Cameron, C., Camolas, J., Can, G., Cândido, A.P.C., Capuano, V., Cardoso, V.C., Carvalho, M.J., Casanueva, F.F., Casas, J.P., Caserta, C.A., Castetbon, K., Chamukuttan, S., Chan, A.W., Chan, Q., Chaturvedi, H.K., Chaturvedi, N., Chen, C.J., Chen, F., Chen, H., Chen, S., Chen, Z., Cheng, C.Y., Chetrit, A., Chiolero, A., Chiou, S.T., Chirita-Emandi, A., Cho, Y., Christensen, K., Chudek, J., Cifkova, R., Claessens, F., Clays, E., Concin, H., Cooper, C., Cooper, R., Coppinger, T.C., Costanzo, S., Cottel, D., Cowell, C., Craig, C.L., Crujeiras, A.B., D’Arrigo, G., D’Orsi, E., Dallongeville, J., Damasceno, A., Damsgaard, C.T., Dankner, R., Dauchet, L., De Backer, G., De Bacquer, D., De Gaetano, G., De Henauw, S., De Smedt, D., Deepa, M., Deev, A.D., Dehghan, A., Delisle, H., Delpeuch, F., Dhana, K., Di Castelnuovo, A.F., Dias-DaCosta, J.S., Diaz, A., Djalalinia, S., Do, H.T.P., Dobson, A.J., Donfrancesco, C., Döring, A., Doua, K., Drygas, W., Egbagbe, E.E., Eggertsen, R., Ekelund, U., El Ati, J., Elliott, P., Engle-Stone, R., Erasmus, R.T., Erem, C., Eriksen, L., De La Peña, J.E., Evans, A., Faeh, D., Fall, C.H., Farzadfar, F., FelixRedondo, F.J., Ferguson, T.S., Fernández-Bergés, D., Ferrante, D., Ferrari, M., Ferreccio, C., Ferrieres, J., Finn, J.D., Fischer, K., Flores, E.M., Föger, B., Foo, L.H., Forslund, A.S., Fortmann, S.P., Fouad, H.M., Francis, D.K., Do Carmo Franco, M., Franco, O.H., Frontera, G., Fuchs, F.D., Fuchs, S.C., Fujita, Y., Furusawa, T., Gaciong, Z., Gafencu, M., Gareta, D., Garnett, S.P., Gaspoz, J.M., Gasull, M., Gates, L., Geleijnse, J.M., Ghasemian, A., Giampaoli, S., Gianfagna, F., Giovannelli, J., Giwercman, A., Goldsmith, R.A., Gross, M.G., Rivas, J.P.G., Gorbea, M.B., Gottrand, F., Graff-Iversen, S., Grafnetter, D., Grajda, A., Grammatikopoulou, M.G., Gregor, R.D., Grodzicki, T., Grøntved, A., Gruden, G., Grujic, V., Gu, D., Guan, O.P., Gudnason, V., Guerrero, R., Guessous, I., Guimaraes, A.L., Gulliford, M.C., Gunnlaugsdottir, J., Guo, X.H., Guo, Y., Gupta, P.C., Gureje, O., Gurzkowska, B., Gutierrez, L., Gutzwiller, F., Halkjær, J., Hardy, R., Kumar, R.H., Hayes, A.J., He, J., Hendriks, M.E., Cadena, L.H., Heshmat, R., Hihtaniemi, I.T., Ho, S.Y., Ho, S.C., Hobbs, M., Hofman, A., Hormiga, C.M., Horta, B.L., Houti, L., Htay, T.T., Htet, A.S., Htike, M.M.T., Hu, Y., Hussieni, A.S., Huu, C.N., Huybrechts, I., Hwalla, N., lacoviello, L., Iannone, A.G., Ibrahim, M.M., Ikram, M.A., Irazola, V.E., Islam, M., Iwasaki, M., Jackson, R.T., Jacobs, J.M., Jafar, T., Jamil, K.M., Jamrozik, K., Jasienska, G., Jiang, C.Q., Joffres, M., Johansson, M., Jonas, J.B., Jørgensen, T., Joshi, P., Juolevi, A., Jurak, G., Jureša, V., Kaaks, R., Kafatos, A., Kalter-Leibovici, O., Kapantais, E., Kasaeian, A., Katz, J., Kaur, P., Kavousi, M., Keil, U., Boker, L.K., Kelishadi, R., Kemper, H.H.C.G., Kengne, A.P., Kersting, M., Key, T., Khader, Y.S., Khalili, D., Khaw, K.T.H., Khouw, I.M.S.L., Kiechl, S., Killewo, J., Kim, J., Kiyohara, Y., Klimont, J., Kolle, E., Kolsteren, P., Korrovits, P., Koskinen, S., Kouda, K., Koziel, S., Kratzer, W., Krokstad, S., Kromhout, D., Kruger, H.S., Kula, K., Kulaga, Z., Kumar, R.K., Kusuma, Y.S., Kuulasmaa, K., Laamiri, F.Z., Laatikainen, T., Lachat, C., Laid, Y., Lam, T.H., Landrove, O., Lanska, V., Lappas, G., Laugsand, L.E., Le Nguyen Bao, K., Le, T.D., Leclercq, C., Lee, Jeannette, Lee, Jeonghee, Lehtimäki, 
T., Rampal, L., León-Munoz, L.M., Lim, W.Y., Lima-Costa, M.F., Lin, X., Linneberg, A., Lissner, L., Litwin, M., Liu, J., Lorbeer, R., Lotufo, P.A., Lozano, J.E., Luksiene, D., Lundqvist, A., Lunet, N., Lytsy, P., Ma, G., Machi, S., Maggi, S., Magliano, D.J., Makdisse, M., Malekzadeh, R., Malhotra, R., Rao, K.M., Manios, Y., Mann, J.I., Manzato, E., Margozzini, P., Markey, O., Marques-Vidal, P., Marrugat, J., Martin-Prevel, Y., Martorell, R., Masoodi, S.R., Matsha, T.E., Mazur, A., Mbanya, J.C.N., McFarlane, S.R., McGarvey, S.T., McKee, M., McLachlan, S., McLean, R.M., McNulty, B.A., Md Yusof, S., Mediene-Benchekor, S., Meirhaeghe, A., Meisinger, C., Mendes, L.L., Menezes, A.M.B., Mensink, G.B.M., Meshram, I.I., Metspalu, A., Mi, J., Michaelsen, K.F., Mikkel, K., Miller, J.C., Miquel, J.F., Mišigoj-Duraković, M., Mohamed, M.K., Mohammad, K., Mohammadifard, N., Mohan, V., Yusoff, M.F.M., Molbo, D., Møller, N.C., Molnár, D., Mondo, C.K., Monterrubio, E.A., Monyeki, K.D.K., Moreira, L.B., Morejon, A., Moreno, L.A., Morgan, K., Mortensen, E.L., Moschonis, G., Mossakowska, M., Mota, J., Motlagh, M.E., Motta, J., Mu, T.T., Muiesan, M.L., Müller-Nurasyid, M., Murphy, N., Mursu, J., Murtagh, E.M., Musa, K.I., Musil, V., Nagel, G., Nakamura, H., Námešná, J., Nang, E.E.K., Nangia, V.B., Nankap, M., Narake, S., Navarrete-Muñoz, E.M., Nenko, I., Neovius, M., Nervi, F., Neuhauser, H.K., Nguyen, N.D., Nguyen, Q.N., Nieto-Martínez, R.E., Ning, G., Ninomiya, T., Nishtar, S., Noale, M., Norat, T., Noto, D., Al Nsour, M., O’Reilly, D., Ochoa-Avilés, A.M., Oh, K., Olayan, I.H., Olinto, M.T.A., Oltarzewski, M., Omar, M.A., Onat, A., Ordunez, P., Ortiz, A.P., Osler, M., Osmond, C., Ostojic, S.M., Otero, J.A., Overvad, K., Paccaud, F.M., Padez, C., Pajak, A., Palli, D., Palloni, A., Palmieri, L., Panda-Jonas, S., Panza, F., Parnell, W.R., Parsaeian, M., Pednekar, M.S., Peeters, P.H., Peixoto, S.V., Pereira, A.C., Pérez, C.M., Peters, A., Peykari, N., Pham, S.T., Pigeot, I., Pikhart, H., Pilav, A., Pilotto, L., Pistelli, F., Pitakaka, F., Piwonska, A., Piwonski, J., Plans-Rubió, P., Poh, B.K., Porta, M., Portegies, M.L.P., Poulimeneas, D., Pradeepa, R., Prashant, M., Price, J.F., Puiu, M., Punab, M., Qasrawi, R.F., Qorbani, M., Bao, T.Q., Radic, I., Radisauskas, R., Rahman, M., Raitakari, O., Raj, M., Rao, S.R., Ramachandran, A., Ramke, J., Ramos, R., Rampal, S., Rasmussen, F., Redon, J., Reganit, P.F.M., Ribeiro, R., Riboli, E., Rigo, F., De Wit, T.F.R., Ritti-Dias, R.M., Rivera, J.A., Robinson, S.M., Robitaille, C., Rodríguez-Artalejo, F., Del Cristo Rodriguez-Perez, M., RodríguezVillamizar, L.A., Rojas-Martinez, R., Rojroongwasinkul, N., Romaguera, D., Ronkainen, K., Rosengren, A., Rouse, I., Rubinstein, A., Rühli, F.J., Rui, O., Ruiz-Betancourt, B.S., Horimoto, A.R.V.R., Rutkowski, M., Sabanayagam, C., Sachdev, H.S., Saidi, O., Salanave, B., Martinez, E.S., Salomaa, V., Salonen, J.T., Salvetti, M., Sánchez-Abanto, J., Sandjaja, Sans, S., Santos, D.A., Santos, O., Dos Santos, R.N., Santos, R., Sardinha, L.B., Sarrafzadegan, N., Saum, K.U., Savva, S.C., Scazufca, M., Rosario, A.S., Schargrodsky, H., Schienkiewitz, A., Schmidt, I.M., Schneider, I.J., Schultsz, C., Schutte, A.E., Sein, A.A., Sen, A., Senbanjo, I.O., Sepanlou, S.G., Shalnova, S.A., Shaw, J.E., Shibuya, K., Shin, Y., Shiri, R., Siantar, R., Sibai, A.M., Silva, A.M., Silva, D.A.S., Simon, M., Simons, J., Simons, L.A., Sjostrom, M., Slowikowska-Hilczer, J., Slusarczyk, P., Smeeth, L., Smith, M.C., Snijder, M.B., So, H.K., Sobngwi, E., Söderberg, S., Soekatri, M.Y.E., Solfrizzi, V., Sonestedt, E., Sørensen, T.I.A., Sorić, M., Jérome, C.S., Soumare, A., Staessen, J.A., Starc, G., Stathopoulou, M.G., Staub, K., Stavreski, B., Steene-Johannessen, J., Stehle, P., Stein, A.D., Stergiou, G.S., Stessman, J., Stieber, J., Stöckl, D., Stocks, T., Stokwiszewski, J., Stratton, G., Strufaldi, M.W., Sun, C.A., Sundström, J., Sung, Y.T., Sunyer, J., Suriyawongpaisal, P., Swinburn, B.A., Sy, R.G., Szponar, L., Tai, E.S., Tammesoo, M.L., Tamosiunas, A., Tang, L., Tang, X., Tanser, F., Tao, Y., Tarawneh, M., Tarp, J., Tarqui-Mamani, C.B., Taylor, A., Tchibindat, F., Thijs, L., Thuesen, B.H., Tjonneland, A., Tolonen, H.K., Tolstrup, J.S., Topbas, M., Topór-Madry, R., Torrent, M., Traissac, P., Trichopoulou, A., Trichopoulos, D., Trinh, O.T.H., Trivedi, A., Tshepo, L., Tulloch-Reid, M.K., Tuomainen, T.P., Tuomilehto, J., Tynelius, P., Tzotzas, T., Tzourio, C., Ueda, P., Ukoli, F.A.M., Ulmer, H., Unal, B., Valdivia, G., Vale, S., Valvi, D., Van Der Schouw, Y.T., Van Herck, K., Van Minh, H., Van Valkengoed, I.G.M., Vanderschueren, D., Vanuzzo, D., Vatten, L., Vega, T., Velasquez-Melendez, G., Veronesi, G., Monique Verschuren, W.M., Viegi, G., Viet, L., Viikari-Juntura, E., Vineis, P., Vioque, J., Virtanen, J.K., Visvikis-Siest, S., 
Viswanathan, B., Vollenweider, P., Voutilainen, S., Vrijheid, M., Wade, A.N., Wagner, A., Walton, J., Mohamud, W.N.W., Wang, M.D., Wang, Q., Wang, Y.X., Wannamethee, S.G., Wareham, N., Weerasekera, D., Whincup, P.H., Widhalm, K., Widyahening, I.S., Wiecek, A., Wilks, R.J., Willeit, J., Wojtyniak, B., Wong, J.E., Wong, T.Y., Woo, J., Woodward, M., Wu, F.C., Wu, J.F., Wu, S.L., Xu, H., Xu, L., Yamborisut, U., Yan, W., Yang, X., Yardim, N., Ye, X., Yiallouros, P.K., Yoshihara, A., You, Q.S., Younger-Coleman, N.O., Yusoff, A.F., Zainuddin, A.A., Zambon, S., Zdrojewski, T., Zeng, Y., Zhao, D., Zhao, W., Zheng, Y., Zhou, M., Zhu, D., Zimmermann, E., Cisneros, J.Z., 2016. Trends in adult bodymass index in 200 countries from 1975 to 2014: A pooled analysis of 1698 population-based measurement studies with 19.2 million participants. Lancet 387, 1377-1396.

https://doi.org/10.1016/S0140-6736(16)30054-X

Esmaeilpour, Z., Shereen, A.D., Ghobadi-Azbari, P., Datta, A., Woods, A.J., Ironside, M., O'Shea, J., Kirk, U., Bikson, M., Ekhtiari, H., 2019. Methodology for tDCS integration with fMRI. Hum. Brain Mapp. https://doi.org/10.1002/hbm.24908

Fregni, F., Orsati, F., Pedrosa, W., Fecteau, S., Tome, F.A.M., Nitsche, M.A., Mecca, T., Macedo, E.C., Pascual-Leone, A., Boggio, P.S., 2008. Transcranial direct current stimulation of the prefrontal cortex modulates the desire for specific foods. Appetite 51, 34-41. https://doi.org/10.1016/j.appet.2007.09.016

Ghobadi-Azbari, P., Jamil, A., Yavari, F., Esmaeilpour, Z., Malmir, N., Mahdavifar-Khayati, R., Soleimani, G., Cha, Y.-H., Shereen, A.D., Nitsche, M.A., Bikson, M., Ekhtiari, H., 2020. fMRI and Transcranial Electrical Stimulation (tES): A systematic review of parameter space and outcomes. medRxiv 2020.06.03.20121202. https://doi.org/10.1101/2020.06.03.20121202

Goldman, R.L., Borckardt, J.J., Frohman, H.A., O’Neil, P.M., Madan, A., Campbell, L.K., Budak, A., George, M.S., 2011. Prefrontal cortex transcranial direct current stimulation (tDCS) temporarily reduces food cravings and increases the self-reported ability to resist food in adults with frequent food craving. Appetite 56, 741-746. https://doi.org/10.1016/j.appet.2011.02.013

Hall, P.A., Lowe, C., Vincent, C., 2017. Brain Stimulation Effects on Food Cravings and Consumption. Psychosom. Med. 79, 839-842. https://doi.org/10.1097/PSY.0000000000000504

Higgs, S., Spetter, M.S., Thomas, J.M., Rotshtein, P., Lee, M., Hallschmid, M., Dourish, C.T., 2017. Interactions between metabolic, reward and cognitive processes in appetite control: Implications for novel weight management therapies. J. Psychopharmacol. 31, 1460-1474. https://doi.org/10.1177/0269881117736917

Hintze, J., 2011. PASS 11. NCSS, LLC. Kaysville, Utah, USA.

Jansen, J.M., Daams, J.G., Koeter, M.W.J., Veltman, D.J., Van Den Brink, W., Goudriaan, A.E., 2013. Effects of non-invasive neurostimulation on craving: A meta-analysis. Neurosci. Biobehav. Rev. https://doi.org/10.1016/j.neubiorev.2013.07.009

Kachooei, M., Ashrafi, E., 2016. Exploring the Factor Structure, Reliability and Validity of the Food Craving Questionnaire-Trait in Iranian adults.

Kekic, M., McClelland, J., Campbell, I., Nestler, S., Rubia, K., David, A.S., Schmidt, U., 2014. The effects of prefrontal cortex transcranial direct current stimulation (tDCS) on food craving and temporal discounting in women with frequent food cravings. Appetite 78, 55-62. https://doi.org/10.1016/j.appet.2014.03.010 
Kim, S., Popkin, B.M., 2006. Commentary: Understanding the epidemiology of overweight and obesity A real global public health concern. Int. J. Epidemiol. https://doi.org/10.1093/ije/dyi255

Kullmann, S., Heni, M., Veit, R., Scheffler, K., Machann, J., Haring, H.U., Fritsche, A., Preissl, H., 2015. Selective insulin resistance in homeostatic and cognitivecontrol brain areas in overweight and obese adults. Diabetes Care 38, 1044-1050. https://doi.org/10.2337/dc14-2319

Larsen, J.K., Hermans, R.C.J., Engels, R.C.M.E., 2012. Food intake in response to food-cue exposure. Examining the influence of duration of the cue exposure and trait impulsivity. Appetite 58, 907913. https://doi.org/10.1016/j.appet.2012.02.004

Lerman, C., Gu, H., Loughead, J., Ruparel, K., Yang, Y., Stein, E.A., 2014. Large-scale brain network coupling predicts acute nicotine abstinence effects on craving and cognitive function. JAMA Psychiatry 71, 523-530. https://doi.org/10.1001/jamapsychiatry.2013.4091

Lowe, C.J., Reichelt, A.C., Hall, P.A., 2019. The Prefrontal Cortex and Obesity: A Health Neuroscience Perspective. Trends Cogn. Sci. https://doi.org/10.1016/j.tics.2019.01.005

Lowe, C.J., Vincent, C., Hall, P.A., 2017. Effects of Noninvasive Brain Stimulation on Food Cravings and Consumption: A Meta-Analytic Review. Psychosom. Med. https://doi.org/10.1097/PSY.0000000000000368

McClelland, J., Kekic, M., Bozhilova, N., Nestler, S., Dew, T., Van Den Eynde, F., David, A.S., Rubia, K., Campbell, I.C., Schmidt, U., 2016. A randomised controlled trial of neuronavigated repetitive transcranial magnetic stimulation (rTMS) in anorexia nervosa. PLoS One 11. https://doi.org/10.1371/journal.pone.0148606

Morris, M.J., Beilharz, J.E., Maniam, J., Reichelt, A.C., Westbrook, R.F., 2015. Why is obesity such a problem in the 21st century? The intersection of palatable food, cues and reward pathways, stress, and cognition. Neurosci. Biobehav. Rev. https://doi.org/10.1016/j.neubiorev.2014.12.002

Mostafavi, S.-A., Khaleghi, A., Mohammadi, M.R., Akhondzadeh, S., 2018. Is transcranial direct current stimulation an effective modality in reducing food craving? A systematic review and meta-analysis. Nutr. Neurosci. 1-13. https://doi.org/10.1080/1028415x.2018.1470371

Mostafavi, S., ... S.K.-I. journal of, 2016, U., 2016. Reliability and validity of the Persian version of Compulsive Eating Scale (CES) in overweight or obese women and its relationship with some body. ncbi.nlm.nih.gov.

Mostafavi, S.A., Akhondzadeh, S., Mohammadi, M.R., Eshraghian, M.R., Hosseini, S., Chamari, M., Keshavarz, S.A., 2017. The reliability and validity of the persian version of three-factor eating questionnaire-R18 (TFEQ-R18) in overweight and obese females. Iran. J. Psychiatry 12, 100-108.

Murdaugh, D.L., Cox, J.E., Cook, E.W., Weller, R.E., 2012. FMRI reactivity to high-calorie food pictures predicts short- and long-term outcome in a weight-loss program. Neuroimage 59, 2709-2721. https://doi.org/10.1016/j.neuroimage.2011.10.071

Odom, J., Zalesin, K.C., Washington, T.L., Miller, W.W., Hakmeh, B., Zaremba, D.L., Altattan, M., Balasubramaniam, M., Gibbs, D.S., Krause, K.R., Chengelis, D.L., Franklin, B.A., McCullough, P.A., 2010. Behavioral predictors of weight regain after bariatric surgery. Obes. Surg. 20, 349-356. https://doi.org/10.1007/s11695-009-9895-6

Oldfield, R.C. \%J N., 1971. The assessment and analysis of handedness: the Edinburgh inventory 9, 97- 
113.

Pierpaoli, C., Basser, P.J., 1996. Toward a quantitative assessment of diffusion anisotropy. Magn. Reson. Med. 36, 893-906. https://doi.org/10.1002/mrm.1910360612

Rogers, P.J., Hill, A.J., 1989. Breakdown of dietary restraint following mere exposure to food stimuli: Interrelationships between restraint, hunger, salivation, and food intake. Addict. Behav. 14, 387397. https://doi.org/10.1016/0306-4603(89)90026-9

Ronzio, R., 2003. The encyclopedia of nutrition and good health.

Sahebi, A., Asghari, M., Salari, R., 2005. Validation of depression anxiety and stress scale (DASS-21) for an Iranian population.

Simmons, W., Martin, A., Cortex, L.B.-C., 2005, U., 2005. Pictures of appetizing foods activate gustatory cortices for taste and reward. academic.oup.com.

Stice, E., Telch, C.F., Rizvi, S.L., 2000. Development and validation of the eating disorder diagnostic scale: A brief self-report measure of anorexia, bulimia, and binge-eating disorder. Psychol. Assess. 12, 123-131. https://doi.org/10.1037/1040-3590.12.2.123

Team, R., 2013. R: A language and environment for statistical computing.

Thielscher, A., Antunes, A., Saturnino, G.B., 2015. Field modeling for transcranial magnetic stimulation: A useful tool to understand the physiological effects of TMS?, in: Proceedings of the Annual International Conference of the IEEE Engineering in Medicine and Biology Society, EMBS. Institute of Electrical and Electronics Engineers Inc., pp. 222-225. https://doi.org/10.1109/EMBC.2015.7318340

Uher, R., Yoganathan, D., Mogg, A., Eranti, S. V., Treasure, J., Campbell, I.C., McLoughlin, D.M., Schmidt, U., 2005. Effect of left prefrontal repetitive transcranial magnetic stimulation on food craving. Biol. Psychiatry 58, 840-842. https://doi.org/10.1016/j.biopsych.2005.05.043

Van den Eynde, F., Claudino, A.M., Mogg, A., Horrell, L., Stahl, D., Ribeiro, W., Uher, R., Campbell, I., Schmidt, U., 2010. Repetitive Transcranial Magnetic Stimulation Reduces Cue-Induced Food Craving in Bulimic Disorders. Biol. Psychiatry 67, 793-795. https://doi.org/10.1016/j.biopsych.2009.11.023

Van Den Eynde, F., Guillaume, S., Broadbent, H., Campbell, I.C., Schmidt, U., 2013. Repetitive transcranial magnetic stimulation in anorexia nervosa: A pilot study. Eur. Psychiatry 28, 98-101. https://doi.org/10.1016/j.eurpsy.2011.06.002

Volkow, N.D., Wang, G.J., Baler, R.D., 2011. Reward, dopamine and the control of food intake: Implications for obesity. Trends Cogn. Sci. https://doi.org/10.1016/j.tics.2010.11.001

Windhoff, M., Opitz, A., Thielscher, A., 2013. Electric field calculations in brain stimulation based on finite elements: An optimized processing pipeline for the generation and usage of accurate individual head models. Hum. Brain Mapp. 34, 923-935. https://doi.org/10.1002/hbm.21479 